\title{
When visual search functions look like item recognition functions*
}

\author{
MARIANNE W. KRISTOFFERSON \\ Psychiam Department. Mchaster Unilersitv, Hamilton. Ontario, Canada \\ and \\ MARY GROEN and ALFRED B. KRISTOFFERSON \\ Psycholog. Department, McMaster Lniversity, Hamilton, Ontario, Canada
}

\begin{abstract}
Visual search data were collected from six Ss on three target set sizes on each of 30 days. Error level was low, and items assigned to memory sets were nonnested and changed from session to session. For each $S$, the same item sometimes required a positive and sometimes a negative response (response inconsistency). Combining data over Ss and over successive 6-day blocks, visual search rates as a function of target set size were found to be linear for each of the five 6-day blocks. The slopes of the above functions (memory search time) did not differ significantly over the final four 6-day blocks. and averaged approximately $.500 \mathrm{sec}$ per six-character item. These results are qualitatively very similar to results obtained from item recognition studies when error level, memory set structure, degree of response consistency. and practice are handled in the same way in that task. The significantly lower slope obtained on the first 6-day block is shown to be consistent with a speed-accuracy tradeoff interpretation when error rate is expressed per unit of processing time (percent errors/set size). Over the final three 6-day blocks, where all important parameters of the data were highly stable, the intercepts of the memory search functions were found to closely approximate zero, averaging $.0068 \mathrm{sec}$. From this finding, along with the finding that the memory search functions are linear, it is inferred that visual search time is determined entirely by memory search time, or by memory search time and other processes which increase linearly with set size, under the conditions of this experiment. The estimate of memory search time (approximately $83 \mathrm{msec} / \mathrm{character}$ ) obtained using this visual search procedure is much slower than that obtained using the item recognition procedure (approximately $35-40 \mathrm{msec} / \mathrm{character}$ ). An explanation for this difference is proposed.
\end{abstract}

From a series of previous studies (Kristofferson, $1972 a, b, c)$, it appears that the variables sufficient to control and predict the effect of the number of items in the memory set (the positive set size or the target set size) on item recognition time and visual search time are: (1) error level, (2) positive set structure, (3) degree of response consistency, and (4) practice.

In the item recognition task, the $S$ must respond "yes" or "no," by pressing the appropriate one of two keys, to indicate whether a presented test item is or is not a member of a predefined positive set of items. The items are usually digits or letters. The size of the positive set is varied, and response latencies provide the basic data. Item recognition time (response latency) increases as positive set size increases (the item recognition function). The function is linear and has the same slope for both positive and negative responses. Typically, item recognition time is increased by approximately $35-40 \mathrm{msec}$ for each additional item assigned to the positive set. This description of the item recognition function is accurate only for a particular combination of the variables listed above: (1) error level is low, errors occurring on less than $5 \%$ of the trials; (2) in a given session, there is either no overlap or only partial overlap

*This research was supported in part by the Medical Research Council of Canada under Grant 3743 and in part by the Addiction Research Foundation of Ontario. A part of this study was submitted by one of the authors (M.G.) as a thesis in partial fulfiliment of the Degree Honours Bachelor of Arts (Psychology), McMaster University, 1971. The authors wish to thank Linda Corsun and Carol Timusk for their assistance in data collection and analysis. of items assigned to each positive set size (nonnested sets), and the assignment of items to positive sets is changed for each experimental session; and (3) the same item sometimes requires a positive response, sometimes a negative one, for each $\mathrm{S}$ (response inconsistency). It has been shown that under this set of conditions, practice does not influence the effect of set size (Kristofferson, 1972a). The function remains linear, and its slope does not change over the course of prolonged practice.

Characteristics of the item recognition function are markedly altered when error level is held low, but (1) each positive set contains all the items also contained in smaller sets (nested sets), and positive sets are constant throughout the experiment, and (2) each item in the total stimulus set consistently requires only a positive or only a negative response (response consistency). The item recognition function in this case is not linear, but negatively accelerated. The set size effect decreases over the course of practice. And, the effect of set size is greater for positive trials than for negative trials (Kristofferson, 1972c).

In the visual search task, the $S$ searches through a list of 50 alphanumeric items of six characters each to locate any one of a set of memorized characters. The size of the memory or positive set is varied. The location of the one positive character in each list changes from trial to trial. Time required to locate the target character is recorded. A number of trials are run for each positive set size. Response latencies are plotted against the number of the line (1-50) on which the positive character occurs, and lines are fit by the method of least squares. The slopes of these lines provide the basic data of the 
experiment and represent the time needed to scan over each nontarget item independently of response factors (visual search time). Visual search time increases as the target set size increases (the search function) early in practice, but, with prolonged practice, visual search time becomes independent of set size under some conditions (Neisser, Novick, \& Lazar, 1963; Wattenbarger, 1968). These conditions are: (1) a high error level, errors occurring on approximately $20 \%$ of the trials, (2) the use of constant and nested target sets, and (3) response consistency.

When a low error criterion is imposed on the above set of conditions, the search function is altered (Kristofferson, 1972b: Wattenbarger, 1968). The search function is negatively accelerated throughout practice. The effect of target set size diminishes with prolonged practice, but stabilizes at a small value.

Thus, under conditions of low error level, constant and nested positive sets, and response consistency, the effects of prolonged practice upon the set size effect in visual search and item recognition have been found to be qualitatively very similar.

The present experiment seeks to show in a still more convincing way that the manipulation of these variables (practice, error level, response consistency, and positive set structure) is sufficient to control and predict the set size effect. In this experiment, visual search performance is investigated over a period of prolonged practice where error level is low, target sets are nonnested, and there is response inconsistency both within and between sessions for each S. That is, each of these four variables is handled in the visual search task as they have been in those item recognition studies which have produced evidence of linear item recognition functions, equality of slopes, obtained from positive and negative trials, and stability of the effect of set size over practice. The prediction is made that the memory search function (that function relating target set size and visual search time) will be linear throughout the course of practice, and the slope of the memory search function will remain constant.

It should be pointed out that in this visual search task Ss search for the "presence" of a memorized character(s). Therefore, visual search times reflect the speed with which Ss scan through list items when the list items do not contain a memorized character(s). Thus, when the effect of set size on visual search time is examined, it is analogous to examining the effect of set size on item recognition performance when only the trials requiring a negative response are considered.

\section{METHOD}

\section{Stimulus Materials}

Each stimulus list contained 50 items. and each item was a string of six letters or letters and numbers chosen randomly from a pool of 22 characters (ACDEFGHIJMOTUVWXY23578) with the restriction that no character occur more than once within an itam.
Separate sets of lists were prepared for each of three target set sizes, 1. 2, and 4. and for each experimental session. For each experimental session separately, 7 characters were drawn randomly without replacement from the pool of 22 characters to compose the three target sets for that experimental session. One character was drawn irom the subset of 7 and was assigned to be the target for the $S=1$ target set size condition. From the remaining 6 characters, 2 characters were drawn randomly to compose the $S=2$ target set size condition. The $S=4$ target set was composed of the remaining 4 characters. Only 1 target character appeared on each list. Thus, each list contained 49 items of context only (nontarget characters) and 1 item of 5 context plus 1 target character. The target's position was randomized over the 300 possible positions, with the restriction that for a given target set size, it could not appear on the same line $(1-50)$ more than once within a given session. For target sets of size greater than 1 ( 2 and 4 ), each character within the target set was equally likely to occur as the target.

For each target set size condition. the context consisted of all the characters from the pool of 22 which were not in the current target set. Thus, when the target set was composed of only 1 character, the context consisted of the remaining 21 nontarget characters. For target set sizes of 2 and 4, context characters consisted of the remaining 20 and 18 nontarget characters, respectively. Thus, on any given experimental day, response requirements were consistent for any given character within a single target set size condition, but inconsistent between target set size conditions. That is, for example, if the letter " $\mathrm{C}$ " was the target character when the set size was 1 . " $\mathrm{C}$ " would appear as a nontarget character in lists prepared for target set sizes of 2 and 4.

Each list was internally generated by an IBM 6400 computer and automatically printed out in a single column, six rows to the inch, on pages of unlined paper, with eight 50 -item lists on each page. Three pages, or 24 lists, were prepared for each target set size condition for each session or experimental day. Since the $S$ was unlikely to search all 24 lists within a target set size condition (see procedures section), the frequency of targets actually seen was not necessarily equal.

\section{Subjects}

Six McMaster University students, three males and three females, who were all naive as to the experimental task, served as Ss. They were paid $\$ 2$ an hour for their participation.

\section{Apparatus and Procedure}

A box with a sloping top and a rectangular opening was used to display the stimulus materials. The opening, which was slightly wider and longer than a single 50 -item stimulus list. was covered with a metal shield which could be released to expose the entire list by pressing a button positioned on the ledge at the base of the face of the apparatus. To initiate a trial. the $S$ pressed the button with his nonpreferred hand, which caused the shield to drop and simultaneously activated a Standard Electric timer. Also on this shelf were holders for a removable metal stylus that housed an ordinary felt-tipped marking pen. When the stylus was lifted from the holder, the timer was stopped and simultaneously a second timer was started. The $S$ was instructed to position the fingers of his preferred hand on the pen prior to initiating a trial. to lift the pen as soon as he saw the target, and to mark the target immediately with the pen. When the pen made contact with the clear plastic sheet covering the stimulus list. it terminated the sccond timer and left a mark on the plastic cover. The equipment was designed to accommodate both left- and right-handed $S$ s.

After each trial. the search time and the time required to move the stylus from its holder to mark the target were sach recorded along with the number of the line on which the target occurred. The shield was then raised by the $\mathrm{l}$. the now stimulus 
list was positioned in the display opening. and the $\mathrm{S}$ was given an auditory ready signal to indicate that he could initiate the next trial.

If the $S$ failed to find the target after scanning through the list, no response times were recorded and that trial was recorded as a false-negative error and an additional list was presented. Similarly, if a false-positive error was made, the search time was not used and a new list was presented as a replacement. A false-positive error was recorded on a trial if any one of the following occurred: (a) the $S$ lifted the stylus from the holder, indicating that he had located the target, and then failed to mark the target; (b) the S lifted the stylus from the holder and there was a delay of $3 \mathrm{sec}$ or more before he marked the target; or (c) the $\mathrm{S}$ marked the target within this time limit but the mark was placed three or more lines above or below the item containing the target.

At the start of each condition, the characters forming the target set for that set size condition were read to the S. A card with these characters typed on it was placed on a ledge at eye level and to the right of the top of the display window so that the $S$ could refer to it throughout the trials on that condition.

\section{Experimental Design}

In each pf 30 successive daily sessions (weekends excluded), each $S$ performed on each of the three target set size conditions $(S=1, S=2, S=4)$ until he produced, in addition to the three practice trials for each condition, 12 errorless trials for each condition for which search times were obtained to allow for the calculation of scanning rate. All trials for a given set size were blocked and the order of these blocks was randomized. Over each successive 6 days. each $S$ received each of the six possible orderings of conditions.

The $S$ s were instructed in the following manner: "This experiment is concerned with visual search. You will be searching through lists of alphanumeric characters to locate any of the target characters which are appropriate for that trial or condition. For example, you might be told to search the list for any of the following characters: FTZU-only one of these characters will be present in any one list and its position will be unpredictable from one list to the next. There will be one target character in every list and its position will be random. We will be measuring the speed with which you can locate the target."

The procedure and the apparatus were then explained to the $S$, and the instruction continued in this way:

"As soon as the shield drops, start searching through the list. Always search from the top down. Try not to recheck preceding lines. You are allowed to search through the list only one time. If you come to the bottom of the list and have not located the target, tell me and I will set up a new trial. Of course, on most trials you should locate the target. Do not be alarmed if you miss the target on a few trials, but you should keep the number of these errors as low as possible. As soon as you find the target, lift the pen from its holder and mark the target with it. Never lift the pen until you have seen the target.

"Speed is important-you should scan the list and mark the targets as quickly' as possible. You do not have to be highly accurate in marking the target but the mark should be either on a line with the target character or on the line immediately above or below it. After you have marked the target, please put the pen back into the holder so that I can set up the next trial. Do you have any questions?

"For the first group of trials the character(s) making up the target set is(are)

During the first few sessions, each $S$ was frequently reminded that he should scan through the lists as quickly as possible while maintaining high accuracy. On the second day of the experiment, he was told that his errors should average no more than 2 per day over all the blocks. Daily results were posted on individual graphs as additional incentive to improve both speed

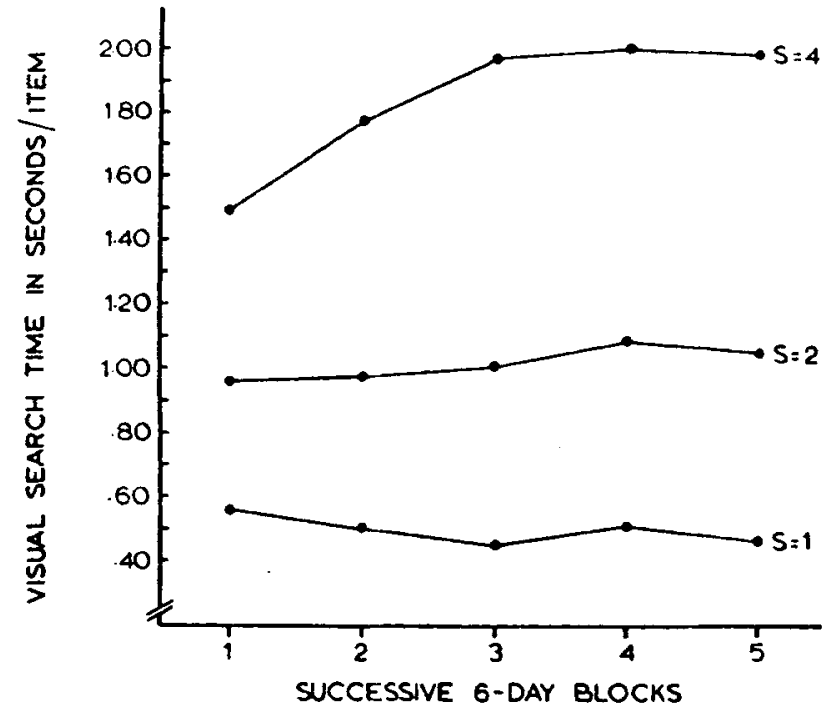

Fig. 1. Visual search time in seconds/item as a function of successive 6-day blocks plotted separately for target set sizes of 1,2 , and 4 .

and accuracy. For each session and each set size condition separately, the 12 correct search times were plotted against the line positions of their respective target charachters and the best-fitting least squares straight line was fit to these data. The slope of this line is interpreted as the time taken to scan a single item in the list. The arithmetic mean of the slopes for the three individual conditions was posted daily for the Ss, as was the total number of errors made in each session.

\section{RESULTS}

A single measure of time per item scanned was determined from each set of 12 search times and their related target positions by the method described by Neisser et al (1963). Equations for the least squares linear functions relating response latencies and target positions were calculated for each $S$, each set size condition, and each day separately. Isolated points which deviated markedly from the others were deleted by the method described by Neisser et al (1963). Slope values obtained from these equations, which represent the time required to scan over each nontarget item independently of response factors, were combined and averaged over Ss for each successive block of 6 days and each target set size separately.

In Fig. 1, mean time-per-item scanned (visual search time) in seconds averaged over all Ss is shown plotted against successive 6-day blocks of trials for each of the three target set size conditions separately. An A by B by $S$ ANOVA was performed to determine the effects of target set size and practice on visual search time. There was a significant main effect of target set size, $F(2,10)=$ $56.60, \mathrm{p}<.0001$. Visual search time increased as a positive function of target set size. The main effect of practice was not significant, $F(4,20)=1.39, \mathrm{p}>.25$. A significant interaction between target set size and 6-day blocks was found, $F(8,40)=6.05, p<.0001$. Duncan's 


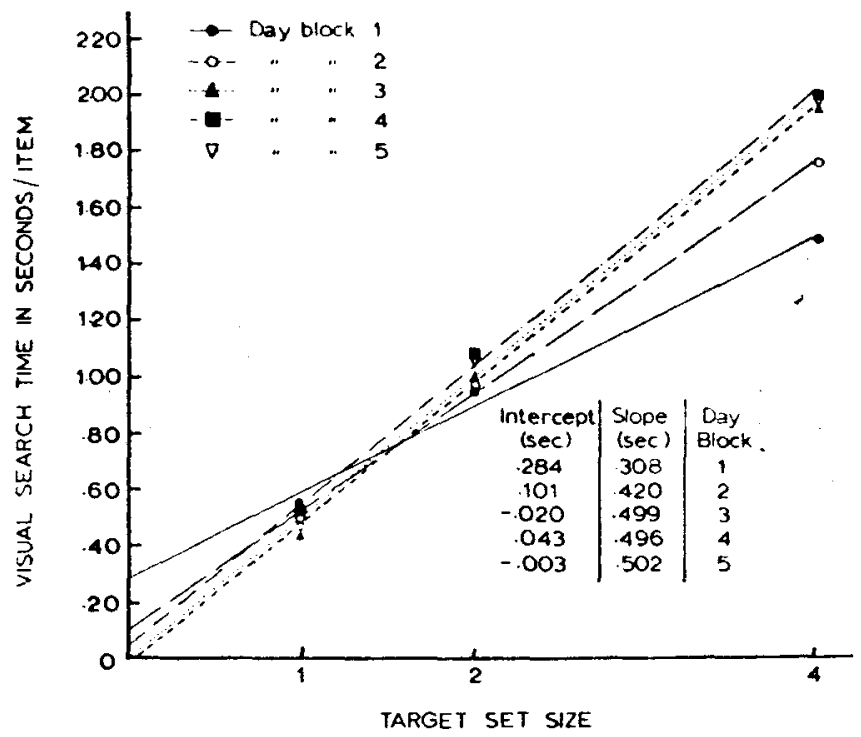

Fig. 2. Visual search time in seconds/item as a function of target set size plotted separately for each successive 6-day block with lines fit by least squares linear regression.

range test applied to these means revealed that for each of the 6-day blocks, visual search time for a target set of 4 was significantly $(\mathrm{p}<.01)$ greater than for a target set of 2 , and visual search time for a target of 2 was significantly greater $(\mathrm{p}<.01)$ than for a single target. This test also showed that visual search times did not change significantly over 6-day blocks when the target set was composed of 1 or of 2 items. The interaction is attributable to the finding that when the target set was composed of 4 items, visual search time was found to change significantly with practice. For this set size, the visual search time for the first 6-day block was significantly faster $(p<.01)$ than for each of the succeeding 6-day blocks; and the visual search time for the second 6-day block was significantly faster $(.01<\mathrm{p}$ $<.05$ ) than for each of the succeeding 6-day blocks.

In Fig. 2, the same data are presented as in Fig. 1, but with a reversal of the roles of dependent variable and parameter. That is, means of visual search times for the group of Ss are plotted as a function of target set size for each successive 6-day block separately in Fig. 2. The percent of variance accounted for by linear regression was determined for each of these five sets of data, and was found to range from $98.9 \%$ to $99.8 \%$. Since these data are clearly well described by linear functions, the slope of each function was determined by the method of least squares in order to obtain measures of memory search time in seconds per item. Intercept and slope values for these functions are also shown in Fig. 2. An A by $B$ by $S$ ANOVA was run to determine whether memory search time (slope) changed over the course of practice. There was a significant main effect of 6-day blocks, $F(4,20)=7.462, p<.001$. Duncan's multiple range test showed that the slope obtained for the first 6-day block was significantly $(\mathrm{p}<.01)$ smaller (memory search time was faster) than the slopes obtained on each of the other 6-day blocks. After the first 6-day block, the slopes did not differ significantly.

For each $\mathrm{S}$, eaci target set size and each successive 6-day block separately, the percent of errors (false positive and false negative combined) was calculated. Percent errors as a function of successive 6-day blocks are plotted separately for each target set size in Fig. 3 . An A by B by S ANOVA was run to determine whether percent of errors differed (a) over the course of practice, and (b) among different target set sizes. There was a significant main effect of target set size, $F(2,10)=$ $30.64, \mathrm{p}<.0002$. The percent of errors increased as target set size increased. There was also a significant main effect of practice, $F(4,20)=9.504, p<.0003$, with percent errors decreasing over the course of practice. The interaction of Target Set Size by 6-Day Blocks was significant, $F(8,40)=9.968, p<.0001$. Comparison of means by using Duncan's multiple range test showed that over all 6-day blocks, significantly $(p<.01)$ more errors were made when the target set was of Size 4 than when the target was only one item. After the first 6-day block, percent of errors for target sets of Sizes 2 and 1 did not differ significantly. For the first three 6-day blocks, percent errors for a target set size of 4 was significantly greater than for a target set size of 2 $(\mathrm{p}<.01)$. On the fourth 6-day block, there was no significant difference in percent errors for these two target set sizes. And, finally, on the fifth 6-day block, percent errors for a target set size of 4 was again significantly $(p<.05)$ greater than for a target set size of 2 . Duncan's multiple range test also showed that for a target set size of 1 , there were no significant differences in the percent of errors over the five successive 6-day blocks. For a target set size of 2 , there was a significantly greater percent of errors on the first 6-day block when compared with each of the subsequent 6-day blocks $(p<.01)$. For a target set size of 4 , the percent of errors on the first 6-day block was significantly $(p<.01)$ greater than for each of the subsequent 6-day

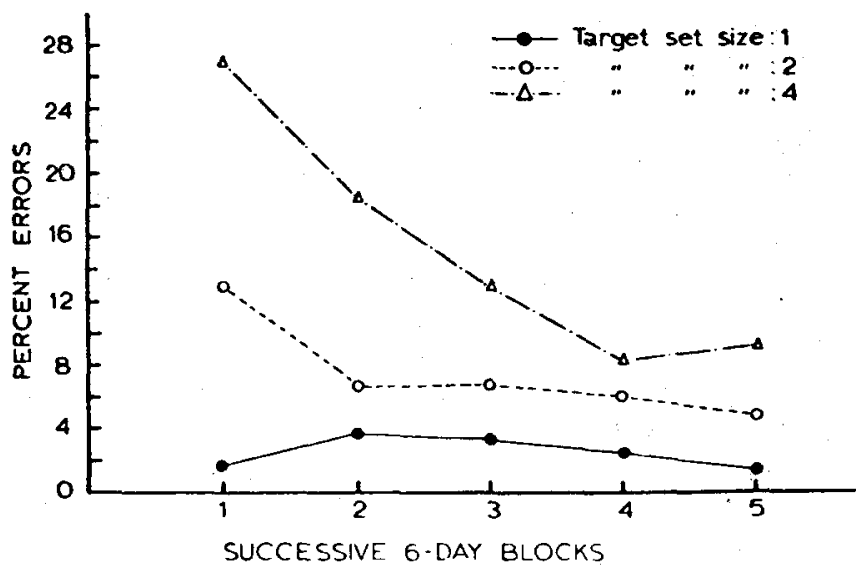

Fig. 3. Percent errors as a function of successive 6-day blocks shown separately for each target set size. 
blocks; percent errors on the second 6-day block was significantly $(p<.01)$ greater than for each of the subsequent 6-day blocks; and percent of errors on the third 6-day block was significantly greater $(p<.05)$ than on the fourth 6-day block.

\section{DISCUSSION}

In general, the results are consistent with those predicted. Memory search functions were found to be well described as linear throughout the course of practice. The slopes of these functions, with the exception of the slope obtained from the first 6-day block, were found not to differ significantly. Over the final three 6-day blocks, visual search time was found to be stable for each of the positive set sizes, which strongly suggests that further practice would not affect the memory search time. On the basis of these findings, it is concluded that under conditions of low error level, nonnested positive sets, and response inconsistency, the effect of set size and the effect of practice on the set size effect as determined from visual search performance is qualitatively very similar to the effect of set size and the effect of practice on the set size as determined from item recognition performance.

It is unusual for skilled performance to slow down with practice. Yet, visual search time per item for the set size of 4 was found to be significantly faster for the first 6-day block than for subsequent 6-day blocks. Consequently, memory search time was found to be significantly faster for the first 6-day block.

In this task, the $\mathbf{S}$ has the option of trading speed for accuracy; further, the $S$ may adopt a different balance between speed and accuracy for different positive set sizes. The error level over the first 6-day block was high, averaging 14\%; and, more importantly, there was a marked increase in error level as a function of positive set size (errors occurred on $2 \%, 13 \%$, and $27 \%$ of the trials for the set sizes of 1,2 , and 4 , respectively). Thus, it seems apparent that the significantly faster memory search time obtained over the early days of the experiment is due to a gain in speed for the larger set sizes at the cost of increased errors.

This very general and unspecified model of the speed-accuracy tradeoff relationship is probably sufficient to account for the single departure obtained from the predicted results, and thus to support the validity of the general conclusions drawn.

However, because it does appear to be the case that Ss can and do trade speed for accuracy in this task, it is important to specify the mechanism by which this is accomplished. In most experiments where the visual search or the item recognition paradigm is used, an attempt is made to equalize the incidence of errors across all positive set sizes, the implicit assumption being that if this attempt is successful then speed and accuracy are balanced in the same way for each set size. But this is not the only possible assumption, and it is perhaps not

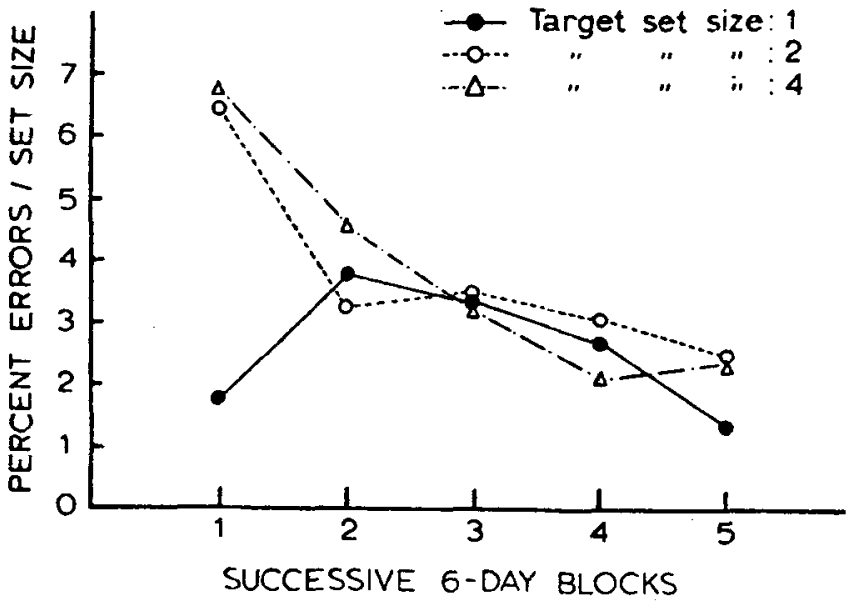

Fig. 4. Percent errors/set size as a function of successive 6-day blocks shown separately for each target set size.

the most reasonable one to make.

It is possible, theoretically, to separate errors made in these tasks (visual search and item recognition) into two types: (1) errors generated in any stage of processing other than the memory search stage, and (2) errors resulting from faulty or incomplete processing within the memory search stage. If errors of the second type occur, errors might be expected to increase in direct proportion to set size. This supposition assumes that speed and accuracy are balanced in the same way for each set size, and is restricted to the case where the number of units involved in memory search are equal to the size of the positive set. This would be the case, even though errors of the first type also occur. Since, in these tasks, units of processing required for all stages of processing other than the memory search stage are assumed to be independent of set size, Type 1 errors would be expected to occur with equal frequency for all set sizes when speed and accuracy are balanced in the same way for each set size.

It follows from this reasoning that if it were found that the percent of errors for a given set size divided by the number of characters in that target set (percent errors/set size) were the same for all set sizes, then that finding would be evidence that the balance between speed and errors is the same at all set sizes.

Can this model of the speed-accuracy tradeoff relationship further clarify the results obtained from the present experiment? Percent errors/set size are shown plotted separately for each target set size as a function of successive 6-day blocks in Fig. 4. An A by B by S ANOVA applied to these data resulted in showing no significant main effect of target set size, $F(2,10)=$ $2.616, p>.10$; a significant main effect of practice, $F(4,20)=4.264, p<.02$; and a significant interaction of Target Set Size by 6-Day Blocks, $F(8,40)=3.392$, $\mathrm{p}<.005$. Individual comparisons between means revealed that, with the exception of the first 6-day block, percent errors/set size did not differ significantly 
among the three positive set sizes. On the first 6-day block, percent errors/set size for set sizes of 4 and 2 were significantly greater $(p<.01)$ than for a set size of 1.

Interpreted within the framework of the model of the speed-accuracy tradeoff relationship proposed, these results lead to the conclusion that for each of the final four 6-day blocks, the balance between speed and accuracy did not differ significantly for the different target set sizes. On the first 6-day block, this balance was not the same for all positive set sizes: for the larger set sizes, more weight was put on speed than on accuracy, as compared to this balance for the set size of 1 . Analyzing errors in this way points up a consistency between changes over 6-day blocks for memory search time and percent errors/set size. On those 6-day blocks (the second through fifth) where the same balance was achieved between speed and errors within each of the target set sizes, memory search time was found to be stable. On the first 6-day block, where greater emphasis was placed on speed for the larger set sizes relative to the set size of 1 , a significantly faster memory search time was obtained.

Over the final three 6-day blocks, visual search time was stable across all three set sizes; memory search times were therefore virtually identical for these three 6-day blocks. Further, percent errors/set size obtained for each of the three set sizes did not differ significantly and the percent errors/set size averaged over set sizes did not change significantly.

An unexpected, but very interesting, finding is that for each of the final three 6-day blocks the intercepts of the memory search functions very closely approximate zero. For the third, fourth, and fifth 6-day blocks, intercept values were $-.020, .043$, and $-.003 \mathrm{sec}$, respectively. If data over these final 18 days of the experiment are averaged together, the intercept of the memory search function is found to be $.0068 \mathrm{sec}$.

This finding, together with the finding that the memory search functions are linear, leads to either of two inferences: (1) visual search time is determined entirely by memory search time, i.e., visual search time is an absolute measurement of memory search time on a ratio scale, and memory search is continuous throughout visual search; or (2) visual search time is determined entirely by memory search time and other processes which also increase in duration in direct proportion to target set size, i.e., visual search time is an absolute measure of these processes on a ratio scale, and these processes are continuous throughout visual search. Both inferences lead to the conclusion that it is improbable that the time required for eye movements and encoding of test stimuli, for example, which might be expected to contribute to visual search time, in fact do. This is the case because there is no reason to expect either of these processes to increase linearly as a function of set size. Finally, then, since processes such as eye movements and stimulus encoding do not contribute at all to visual search time, it must be concluded that these processes are carried out in parallel with that process, or those processes, which determines visual search time.

For each item added to the target set (positive set), the additional time required to search memory for each item in the display was found to average approximately $.500 \mathrm{sec}$ over the final three 6-day blocks. An item is composed of six characters, thus the addition of one item to the target set increases memory search time for a single character in the display by an average of approximately $83 \mathrm{msec}$. The absolute estimate of memory search rate, as obtained by the present visual search procedure, is then much slower than the estimate of memory search rate obtained in item-recognition experiments (approximately $40 \mathrm{msec}$ ).

If the same memory search process is being measured in both types of experiments, it seems inconsistent to maintain that the visual search procedure used here allows direct measurements of memory search time, and then to find that the memory search rate obtained from the visual search procedure is so much slower than that obtained when the item recognition procedure is used. It seems more likely, then, that visual search is determined by memory search and other processes which increase in duration in direct proportion to set size.

Vast differences remain between the present visual search task and the traditional item recognition task. One difference is that in the visual search task, processing goes on continuously, under the conditions of this experiment for an average of about $12 \frac{1}{2} \mathrm{sec}$ on each trial, while in the item recognition task, there is a brief burst of processing activity on each trial. Keeping this difference in mind, an interesting line of speculation has to do with the process by which items composing the positive sets are maintained in active memory and how such a process might differentially affect memory search rate as measured in these two kinds of experiments. It is now widely accepted that at least one function of the active process of rehearsal is to maintain items in short-term memory (or to regenerate decaying traces of item representations in short-term memory). Ss report rehearsal of items composing the positive sets during visual search trials. It seems likely that the time required to carry out a single rehearsal of the positive set items would increase (and probably linearly) as a function of the number of items in the positive set. Thus, visual search time, as measured by this procedure, may reflect some mixture of two processes involved in memory search, one being the serial comparison of the memory items with a display stimulus and the other being the rehearsal of the positive set. In the item recognition task, the $S$ is given a warning signal, and then, after a constant delay, the test stimulus is exposed. Under these conditions, it seems reasonable that the $S$ would rehearse the positive items during the interval between the warning signal and the exposure of the test item, thereby having available in active memory at the time that the test item occurs the items that compose the positive set. 
Thus, it may be that. in the item recognition task, only that aspect of memory search having to do with the serial comparison of the memory items with the display stimuli is measured. This line of speculation could account for the absolute difference between memory search rates obtained from the two different kinds of experiments.

\section{REFERENCES}

Kristofferson, M. W. Effects of practice on character-classification performance. Canadian Journal of Psychology, 1972a, 26, 54-60.
Kristofferson, M. W. Types and frequency of errors in visual search. Perception \& Psychophysics, 1972b, 11, 325-328.

Kristofferson, M. W. When item recognition and visual search functions are similar. Perception \& Psychophysics, 1972c, 12 , 379-384.

Neisser. U., Novick, R., \& Lazar, R. Searching for ten targets simultaneously. Perceptual \& Motor Skills, 1963, 17, 955-961.

Sternberg, S. High-speed scanning in human memory. Science, $1966,153,652-654$.

Wattenbarger, B. L. Speed and accuracy set in visual search performance. Paper presented at the meeting of the Midwestern Psychological A ssociation, Chicago, 1968.

(Received for publication August 28, 1972; revision received March 22, 1973.) 